\title{
Modelling of Thermomechanical Fatigue Stress Relaxation in a Single-Crystal Nickel-Base Superalloy
}

\author{
Daniel Leidermark and Mikael Segersäll
}

\section{Linköping University Post Print}

\section{Tweet}

N.B.: When citing this work, cite the original article.

Original Publication:

Daniel Leidermark and Mikael Segersäll, Modelling of Thermomechanical Fatigue Stress Relaxation in a Single-Crystal Nickel-Base Superalloy, 2014, Computational materials science, (90), , 61-70.

http://dx.doi.org/10.1016/j.commatsci.2014.04.009

Copyright: Elsevier

http://www.elsevier.com/

Postprint available at: Linköping University Electronic Press

http://urn.kb.se/resolve?urn=urn:nbn:se:liu:diva-107983 


\title{
Modelling of thermomechanical fatigue stress relaxation in a single-crystal nickel-base superalloy
}

\author{
Daniel Leidermark ${ }^{*}$, a Mikael Segersäll ${ }^{\mathrm{a}}$ \\ ${ }^{a}$ Department of Management and Engineering, Linköping University, SE-58183 Linköping, \\ Sweden
}

\begin{abstract}
The thermomechanical fatigue (TMF) stress relaxation of the single-crystal nickel-base superalloy MD2 has been analysed and modelled in this paper. Inphase and out-of-phase TMF experiments in the nominal [001], [011] and [111] crystal orientations have been performed. The TMF cycle consists of two loadings each with a $100 \mathrm{~h}$ long hold-time. A simple crystallographic creep model, based on Norton's creep law, has been developed and used in conjunction with a crystal plasticity model. The model takes anisotropy and tension/compression asymmetry into account, where the anisotropic behaviour is based on the crystallographic stress state. The values of the creep parameters in the anisotropic expression were determined by inverse modelling of the conducted TMF experiments, a parameter optimisation were performed. The developed model predicts the stress relaxation seen in the TMF experiments with good correlation.
\end{abstract}

Key words: Single-crystal superalloy, Thermomechanical fatigue, Creep, Stress relaxation, Anisotropy, Parameter optimisation

\section{Introduction}

The market for renewable energy technologies is continuing to grow, and an increase of renewable sources like wind and solar power is expected. Since the production by such sources cannot be actively planned, the grid must be built in such a way that the wind or solar energy can be efficiently received when there is such production, but also so that one can manage over periods where

\footnotetext{
${ }^{*}$ Corresponding author. Tel: $+46(0) 13282791$

Email address: daniel.leidermark@liu.se (Daniel Leidermark)
} 
there is none. In short, this means that the wind and solar energy power must be balanced by other power sources that can be regulated (up and down) on a minute-by-minute basis. This can, for instance, be achieved by gas turbines [1], and an increasing demand for gas turbines designed to stand high numbers of starts and stops can therefore be foreseen. The components in the hot sections of the gas turbine will experience thermomechanical fatigue (TMF) loading, i.e., both thermal and mechanical cycling. Further, during the steady-state condition, also known as hold-time, under service load the components will undergo creep deformation if the temperature is sufficiently high, and the stress state will relax.

The hottest components in a power generating gas turbine are the blades and guide vanes of the first turbine stage. These are mainly produced in the stateof-the-art material, preferable single-crystal nickel-base superalloys which have excellent mechanical properties in very hot environments [2]. These materials will increase the efficiency of the gas turbine as a higher operating temperature can be achieved compared to other materials, which in turn reduces the fuel consumption, leading to reduced environmental effects and lower operating costs [3].

Single-crystal nickel-base superalloys have a face-centered-cubic crystal structure [4], with well defined crystallographic slip planes and slip directions. The material exhibit a well known elastic anisotropy and anomalous plastic behaviour, such as plastic anisotropy and tension/compression asymmetry, which are dependent on the crystallographic structure of the material. As the component is loaded, plastic flow will eventually occur, and the deformation will take place along the crystallographic slip planes, also known as crystal plasticity, see eg. Haupt [5] or Roters et al. [6].

There are two main types of TMF cycles which can affect the components of the gas turbine, the in-phase (IP) TMF cycle and the out-of-phase (OP) TMF cycle. In the IP case the temperature is cycled in-phase with the applied mechanical strain, and in the OP case the temperature is cycled in reverse to the mechanical strain. The typical differences between the two types can be seen in Han et al. [7], where the deformation and damage mechanisms of the first generation single-crystal superalloy SRR99 was investigated for both IP and OP conditions. A display of the two different load cycles and the corresponding hysteresis loops, where one can observe the unsymmetrical shape of the stress-strain curve, can be found. The OP TMF behaviour with hold-time of the single-crystal superalloy CMSX-4 was investigated in Moverare et al. [8], where a practical TMF procedure is described. It is stated that the OP case is typically the one for the hottest region of a component, where a local point might experience compression at the peak temperature, a so called hot-spot.

At comparative low temperatures, at least in the case of gas turbines, $100-$ 
$500^{\circ} \mathrm{C}$, a single-crystal superalloy is not likely to undergo creep deformation. As the components experience a TMF cycle, in which room temperature conditions might be reached, it is thus crucial that the low temperature constitutive behaviour of the material is correctly captured. In the work of Qin and Bassani $[9,10]$ a physically inspired room temperature crystal plasticity model was developed using Schmid and non-Schmid stresses to take the observed anomalous plastic behaviour into account. The model manages to calculate the relative size of the tension/compression asymmetry in the [001], [011] and [111] crystallographic directions. This model was later enhanced by Leidermark et al. [11] to incorporate further non-Schmid stresses to be able to describe the low temperature behaviour of a single-crystal nickel-base superalloy. A comparison with experimentally made tension and compression tests of the single-crystal nickel-base superalloy MD2 were made with good agreement.

At higher temperatures, in the area of approximately $600-800^{\circ} \mathrm{C}$, the singlecrystal material will start to experience time dependent deformation, creep, and in combination with the long hold-times at the steady-state condition resulting in relaxation of the stress state. There are many different types of creep models for single-crystal nickel-base superalloys, Ma et al. [12] proposed a microscopic dislocation model using dislocation glide and climb to account for the creep deformation of the single-crystal superalloy CMSX-4. Another dislocation model is presented in Zhu et al. [13], which couples the alloy chemistry to the creep deformation. A macroscopic viscoplastic model using the Orowan's assumption, that creep strain rate is proportional to the density of the mobile dislocations, has been combined with a rate-independent crystal plasticity approach by Staroselsky and Cassenti [14] to accommodate for the material behaviour of the single-crystal nickel-base superalloy PWA1484 in both LCF and OP TMF tests. In the paper of Ghosh et al. [15] an anisotropic dislocation creep model is described, which includes octahedral and cube slip in combination with a damage parameter that is dependent on the dislocation density. The model is able to predict the anisotropic creep response for a limiting set of experimental data. A phenomenological microscopic-macroscopic model based on rate-dependent crystal plasticity using a damage parameter following Kachanov's creep damage evolution for each slip system has been considered in the work of Vladimirov et al. [16]. The derived model was fit to uniaxial test data and validated for a multiaxial test case where a good agreement with primary, secondary and tertiary creep was obtained. Instead of incorporating the damage on the crystallographic level MacLachlan et al. [17] introduced a global damage tensor derived from the strain rate tensor to describe the creep deformation.

For even higher temperatures, $800-1100^{\circ} \mathrm{C}$, the material is also likely to undergo microstructural changes, so called rafting. Rafting is a time dependent process, that requires some induced plasticity, which gradually degrade the $\gamma / \gamma^{\prime}$ microstructure and affects the mechanical properties of the material, for more details see eg. Epishin et al. [18] and/or Leidermark et al. [19]. 
The objective of this paper is to model the TMF stress relaxation behaviour, creep deformation, observed in the single-crystal nickel-base superalloy MD2. Experiments have been made, where the three crystallographic orientations [001], [011] and [111] have been investigated both in IP and OP TMF. Two TMF cycles each with a hold-time of $100 \mathrm{~h}$ have been applied, from which the creep response of the material is obtained. A new simplistic creep model is developed based on the creep deformation along the crystallographic slip planes. The model uses an anisotropic expression following the basic Norton creep law to define the amount of creep flow in each slip system. The TMF stress relaxation response from the experiments is used in an inverse modelling process (parameter optimisation) in which the creep parameters are determined through curve-mapping with the response from the FE-simulations. From the seen results of the parameter optimisation a good response is obtained, able to describe the anisotropic behaviour of the superalloy.

\section{Experiment}

The chemical composition of the investigated single-crystal nickel-base superalloy MD2 is $\mathrm{Ni}, 5.1 \mathrm{Co}, 6.0 \mathrm{Ta}, 8.0 \mathrm{Cr}, 8.1 \mathrm{~W}, 5.0 \mathrm{Al}, 1.3 \mathrm{Ti}, 2.1 \mathrm{Mo}, 0.1 \mathrm{Hf}, 0.1 \mathrm{Si}$ (in wt.\%). The material was solution heat treated at $1275^{\circ} \mathrm{C}$ for $8 \mathrm{~h}$ followed by a two stage ageing process for $3 \mathrm{~h}$ at $1100^{\circ} \mathrm{C}$ and $24 \mathrm{~h}$ at $850^{\circ} \mathrm{C}$. Six smooth test specimens were machined from cast bars with their longitudinal axes nominally parallel to the [001], [011] and [111] crystal directions, two in each direction. The diameter of the specimens is approximately $6 \mathrm{~mm}$ and the parallel length is $24.58 \mathrm{~mm}$, for more details regarding the geometry of the specimen please see Figure 1. During the casting process a perfect aligned bar is almost impossible to achieve, thus all cast bars have a small misalignment in crystal orientation. The misalignment from the ideal longitudinal (primary) crystal orientations, denoted $\theta$, varied between $1.8^{\circ}$ and $6.2^{\circ}$ for the specimens. The deviation from the ideal secondary crystal orientation ( $\mathrm{SCO}$ ), denoted $\phi$, is of less concern than the primary deviation as the specimens are smooth and round. The nominal secondary crystal orientation can be either the [100], [100], [010] or [010] direction, for the nominal primary crystal orientation [001]. The misalignments and the individual diameters are listed in Table 1 for each specimen and the misalignments are defined according to Figure 2. It is to be pointed out that $\theta$ and $\phi$ are defined with respect to the nominal [001] crystal orientation regardless of primary orientation in the respective test specimens.

The specimens were alternately subjected to either an IP or an OP TMF cycle, i.e. $R_{\varepsilon}=\varepsilon_{\min } / \varepsilon_{\max }$ is equal to 0 and $-\infty$ respectively, where the temperature ranged between $100^{\circ} \mathrm{C}$ and $750^{\circ} \mathrm{C}$ in the loading. The mechanical strain range $\left(\Delta \varepsilon_{m e c h}\right)$ of $1 \%, 0.7 \%$ and $0.6 \%$ were applied for the [001], [011] and [111] crystal orientations, respectively. The difference in applied mechanical strain range 
Table 1: The experimental test specimens misalignment, diameter and applied strain range.

\begin{tabular}{lcccc}
\hline Specimen & $\theta\left(^{\circ}\right)$ & $\phi\left(^{\circ}\right)$ & Diameter $(\mathrm{mm})$ & $\Delta \varepsilon_{\text {mech }}(\%)$ \\
\hline$E_{I P[001]}$ & 1.80 & 30.9 & 6.29 & 1.0 \\
$E_{O P[001]}$ & 6.20 & 40.7 & 6.31 & 1.0 \\
$E_{I P[011]}$ & 43.3 & 0.40 & 6.33 & 0.7 \\
$E_{O P[011]}$ & 43.1 & 0.60 & 6.34 & 0.7 \\
$E_{I P[111]}$ & 51.4 & 39.6 & 6.00 & 0.6 \\
$E_{O P[111]}$ & 53.2 & 42.6 & 6.00 & 0.6 \\
\hline
\end{tabular}

is due to the fact that an initial equivalent inelastic strain $(\sim 0.5 \%)$ is to be present in the specimens for the different crystal orientations at the start of the hold-time. The TMF loading cycle was done in the following sequence: loading from $100^{\circ} \mathrm{C}$ to $750^{\circ} \mathrm{C}$ at a loading rate of $5^{\circ} \mathrm{C} / \mathrm{s}$, a hold-time of $100 \mathrm{~h}$ was applied followed by an unloading with the reversed properties as in the loading stage, this sequence was then applied one more time to achieve two TMF cycles, see Figure 3 for a more detailed overview.

The experimental TMF tests were performed in an Instron servo-hydraulic TMF machine using induction heating and forced air-cooling for rapid temperature changes. The testing machine was carefully aligned to prevent buckling of the specimens. A high-temperature extensometer with a gauge length of $12.5 \mathrm{~mm}$ was used during the tests, and in order to obtain the mechanical strain, the induced thermal strain was subtracted from the total strain. For a more detailed description of the experimental procedure see Segersäll et al. [20].

The reasons why only six test specimens were used in the study were due to the high manufacturing cost and availability of the single-crystal specimens, and consequently the statistical uncertainties in the analysis are bound to increase.

\section{Modelling}

Modelling of the material behaviour has been made in a large deformation context, as the components made of single-crystal material in a gas turbine might experience large deformation when loaded. Subsequently, the following relations have been used in a number of FE-simulations to capture the responses seen in the experimental testing.

When a body is deformed the configuration is changed from the reference to the current configuration which may be described by use of the total deformation gradient $\boldsymbol{F}$, see e.g. Haupt [5]. By introducing an intermediate configuration the total deformation gradient tensor can be multiplicatively split into an elastic 


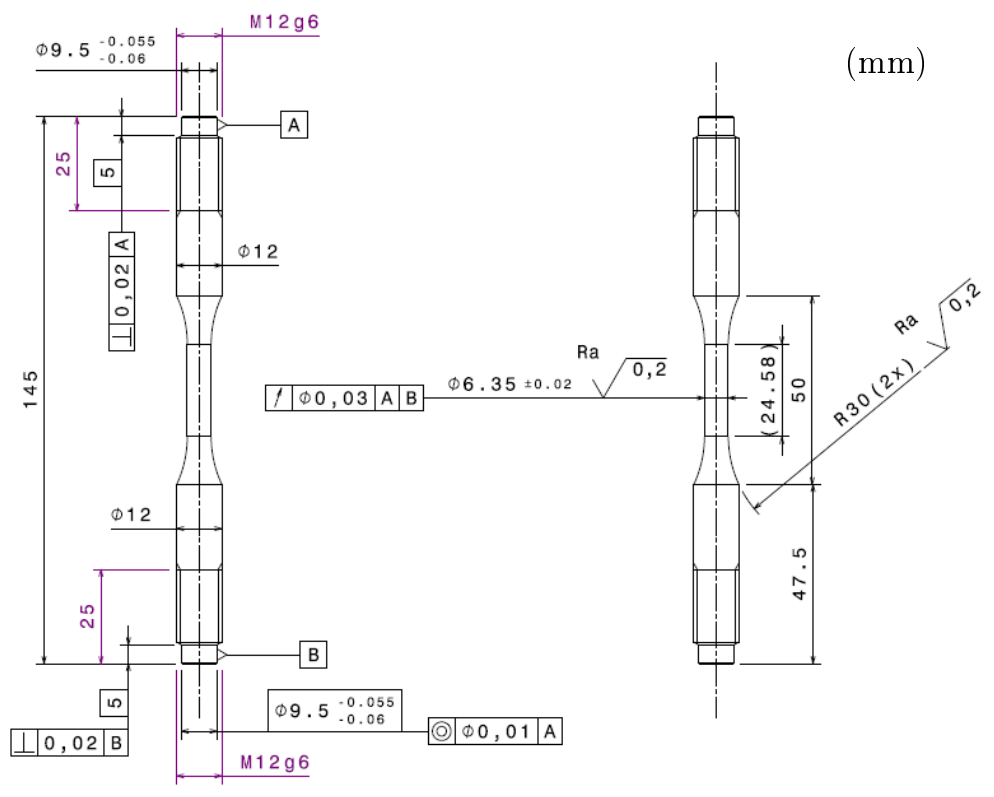

Figure 1: The geometry of the investigated test specimens.

and an inelastic part [21]

$$
\boldsymbol{F}=\boldsymbol{F}^{e} \boldsymbol{F}^{i n}
$$

where the inelastic deformation gradient tensor $\boldsymbol{F}^{i n}$ represents the change between the reference and the intermediate configuration, physically represented by shearing of the lattice, and where the elastic deformation gradient tensor $\boldsymbol{F}^{e}$ represents the deformation done by elastically stretching and rotating the lattice from the intermediate to the current configuration [5]. From the above statement it can be concluded that the velocity gradient tensor can be expressed in the following way

$$
\boldsymbol{L}=\dot{\boldsymbol{F}} \boldsymbol{F}^{-1}=\dot{\boldsymbol{F}^{e}} \boldsymbol{F}^{e^{-1}}+\boldsymbol{F}^{e} \dot{\boldsymbol{F}}^{i n} \boldsymbol{F}^{i n^{-1}} \boldsymbol{F}^{e^{-1}}
$$

where the quantity $\boldsymbol{F}^{i n} \boldsymbol{F}^{i n^{-1}}=\boldsymbol{L}^{i n}$ is the inelastic velocity gradient tensor defined in the intermediate configuration.

In single-crystal nickel-base superalloys the inelastic deformation occurs due to slip on the active slip systems [22], which can be represented by the inelastic 


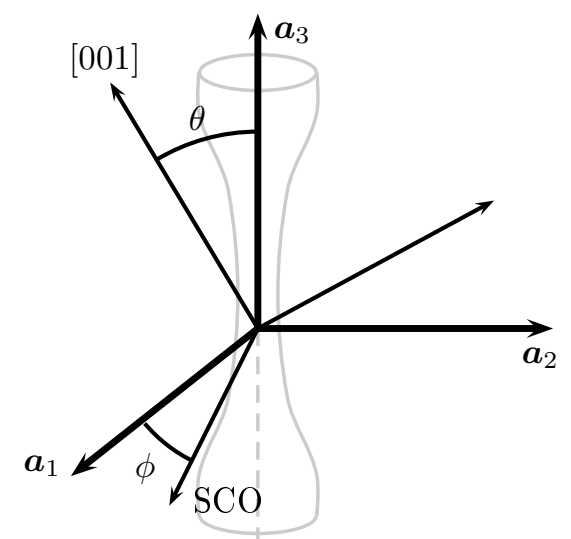

Figure 2: Definition of the specimen misalignments with respect to the nominal primary ([001]) and secondary crystal orientations (SCO).

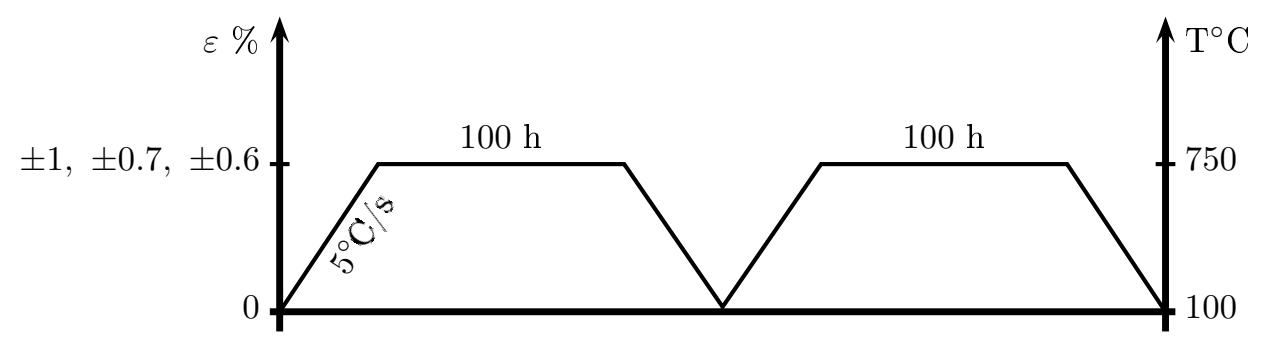

Figure 3: The TMF cycle which was used in the experiments and FE-simulations of this study.

velocity gradient tensor defined in the intermediate configuration accordingly to

$$
\boldsymbol{L}^{i n}=\sum_{\alpha} \dot{\gamma}^{\alpha} \boldsymbol{s}^{\alpha} \otimes \boldsymbol{n}^{\alpha}
$$

where $\dot{\gamma}^{\alpha}$ is the inelastic shear strain rate on slip system $\alpha$, and where $\boldsymbol{s}^{\alpha}$ and $\boldsymbol{n}^{\alpha}$ are the slip and normal directions on the slip plane, respectively, defined in the intermediate configuration. The numerical update of the inelastic deformation gradient tensor can be performed by applying a backward-Euler scheme on the inelastic velocity gradient tensor, thus yielding

$$
\left\{\begin{array}{l}
\Delta t_{n+1} \boldsymbol{L}_{n+1}^{i n}=\Delta \boldsymbol{F}_{n+1}^{i n} \boldsymbol{F}_{n+1}^{i n^{-1}}=\left(\boldsymbol{F}_{n+1}^{i n}-\boldsymbol{F}_{n}^{i n}\right) \boldsymbol{F}_{n+1}^{i n^{-1}}=\boldsymbol{I}-\boldsymbol{F}_{n}^{i n} \boldsymbol{F}_{n+1}^{i n^{-1}} \\
\Delta t_{n+1} \boldsymbol{L}_{n+1}^{i n}=\sum_{\alpha} \Delta \gamma_{n+1}^{\alpha} \boldsymbol{s}^{\alpha} \otimes \boldsymbol{n}^{\alpha}
\end{array}\right.
$$

where $\Delta \gamma_{n+1}^{\alpha}=\Delta t_{n+1} \dot{\gamma}^{\alpha}$ is the incremental inelastic shear strain. By setting the two equations equal and with further manipulations the new updated inelastic deformation gradient tensor $\boldsymbol{F}_{n+1}^{i n}$ for the next iteration step is received 
according to

$$
\boldsymbol{F}_{n+1}^{i n}=\left(\boldsymbol{I}-\sum_{\alpha} \Delta \gamma_{n+1}^{\alpha} \boldsymbol{s}^{\alpha} \otimes \boldsymbol{n}^{\alpha}\right)^{-1} \boldsymbol{F}_{n}^{i n}
$$

A two-step constitutive material model approach is used by sequentially combining creep and plasticity. The problem is solved sequentially by first performing an elastic predictor step, then a creep corrector step is done where the result from the elastic predictor step is taken as initial values. This solution then defines a trial state for the plastic corrector step, which if it is located outside of the yield surface, is incrementally solved back to the yield surface. Note that three possible scenarios might happen; a pure elastic response can be obtained, or the elastic predictor step might end up outside the yield surface and the creep correction takes the stress back to an elastic state, or if the yield function is larger than zero after the creep correction has been performed then the plastic correction step will take the stress back to the yield surface, see Figure 4. Worth pointing out is that the creep flow is considered continuous during the whole process in contrast to the plastic flow. Consequently, as the sequential partitioning of the corrector step between creep and plasticity is present, the inelastic velocity gradient tensor is additively decomposed into a plastic part and a creep part following the work of Staroselsky and Cassenti [14] accordingly to

$$
\boldsymbol{L}^{i n}=\boldsymbol{L}^{p}+\boldsymbol{L}^{c r}
$$

Therefore, the numerical approach for determining the inelastic deformation gradient tensor in each timestep is due to the accumulation of the incremental shear strain in the plastic and creep step, in the following way

$$
\Delta \gamma^{\alpha}=\Delta \gamma^{\alpha, p}+\Delta \gamma^{\alpha, c r}
$$

Thus, to obtain the shear strain rates a flow rule for each of the two steps has to be set up, one for plasticity and one for creep.

(a)

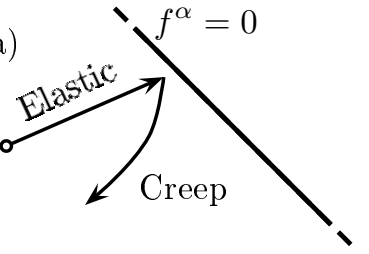

(b)

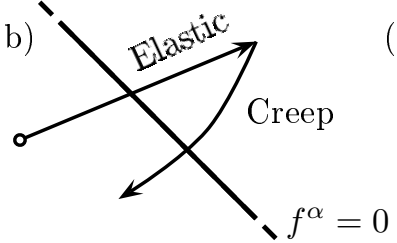

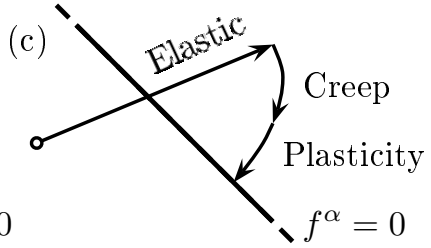

Figure 4: The inelastic deformation gradient is iteratively updated following an elastic trial and creep step, where the trial state might end up either (a) under or (b) above the yield surface, and also followed by a (c) plastic corrector step, if $f^{\alpha}>0$ at the end of the creep step. 


\subsection{Plastic flow}

The crystal plasticity model presented in Leidermark et al. [11] has been used in this work. The model is based on crystal plasticity and takes Schmid as well as non-Schmid stresses into account, thus able to describe the tension/compression asymmetry as well as the plastic anisotropy observed for this material. Twelve unique slip systems are considered, $\alpha=1, \ldots, 12$, resulting in twelve yield functions, one for each slip system. As negligibly small hardening was observed in monotonic loading the following perfect plastic yield functions have been set up

$$
f^{\alpha}=\sigma_{e}^{\alpha}-G_{r}
$$

where $G_{r}$ is the slip resistance on the slip plane, which has the same value for all slip systems, and where the following macroscopic equivalent stresses have been employed

$$
\sigma_{e}^{\alpha}=\left|\tau_{p b}^{\alpha}\right|+\kappa_{1}\left|\tau_{c b}^{\alpha}\right|+\kappa_{2}\left|\tau_{s b}^{\alpha}\right|+\kappa_{3} \tau_{p e}^{\alpha}+\kappa_{4} \tau_{s e}^{\alpha}+\kappa_{5} \sigma_{p n}^{\alpha}
$$

with the following stress components; $\tau_{p b}^{\alpha}$ is the resolved shear stress (Schmid stress) in the close-packed direction of slip system $\alpha$ and the other five $\tau_{c b}^{\alpha}, \tau_{p e}^{\alpha}$, $\tau_{s e}^{\alpha}, \tau_{s b}^{\alpha}$ and $\sigma_{p n}^{\alpha}$ are non-Schmid stresses. These stress components act in different directions on the octahedral, cube and secondary slip planes and have been considered based on inspiration from crystallographic mechanisms occurring on the micro level in the crystal [23]. It is to be noted that the Schmid and nonSchmid stresses, in this modelling context, represent the mean value stresses over a matrix with ordered $\gamma^{\prime}$-particles. Furthermore, $\kappa_{1}$ through $\kappa_{5}$ are weight factors that describe how the non-Schmid stresses affect the yield limit. The first three terms in the equivalent stress expression handle the anisotropy and the last three terms manifest the tension/compression asymmetry. The weight factors and $G_{r}$ are temperature dependent, thus yielding different impacts on the yield criteria as the temperature changes. It can be seen in Leidermark et al. [11] how the weight factors $\kappa_{1}$ through $\kappa_{5}$ and $G_{r}$ are determined by experimentally obtained yield data for a given temperature.

The plastic flow rule of a single-crystal superalloy can be described by shearing along the slip direction, thus the following non-associated flow rule has been adopted

$$
\dot{\gamma}^{\alpha, p}=\dot{\lambda}^{\alpha, p} \frac{\partial g^{\alpha}}{\partial \tau_{p b}^{\alpha}}
$$

where the flow is in the direction of the resolved shear stress (Burgers' vector), hence

$$
g^{\alpha}=\left|\tau_{p b}^{\alpha}\right|
$$

and where, in order to handle rate independent perfect crystal plasticity, the following viscoplastic flow function was used

$$
\dot{\lambda}^{\alpha, p}=\dot{\gamma}_{0}\left\langle\left(\frac{\sigma_{e}^{\alpha}}{G_{r}}\right)^{m}-1\right\rangle
$$


where $\dot{\gamma}_{0}$ and $m$ are regularisation parameters given the values 0.1 and 10 , respectively, in order to obtain the sharp corner of the perfectly plastic behaviour in the stress-strain curve.

\subsection{Creep flow}

The focus in this work is on the modelling of the TMF stress relaxation in the investigated single-crystal material. Hence, the creep model needs to be able to describe the stress relaxation during the long hold-times which the test specimens were exposed to in the experiments. Steady-state secondary creep is considered and modelled, due to the observed TMF stress relaxation behaviour seen in the experiments and from a set of performed uniaxial creep tests of the material in question, which have been reported in Segersäll et al. [20]. At a sufficiently large temperature, dislocation will start to move on the slip planes, yielding a creep flow. A basic assumption is thus, that the creep flow rule will have a similar form as the plastic one, hence a non-associate flow along the slip direction, as in Equation (11), will also be appropriate here

$$
\dot{\gamma}^{\alpha, c r}=\dot{\lambda}^{\alpha, c r} \frac{\partial g^{\alpha}}{\partial \tau_{p b}^{\alpha}}=\dot{\lambda}^{\alpha, c r} \operatorname{sgn}\left(\tau_{p b}^{\alpha}\right)
$$

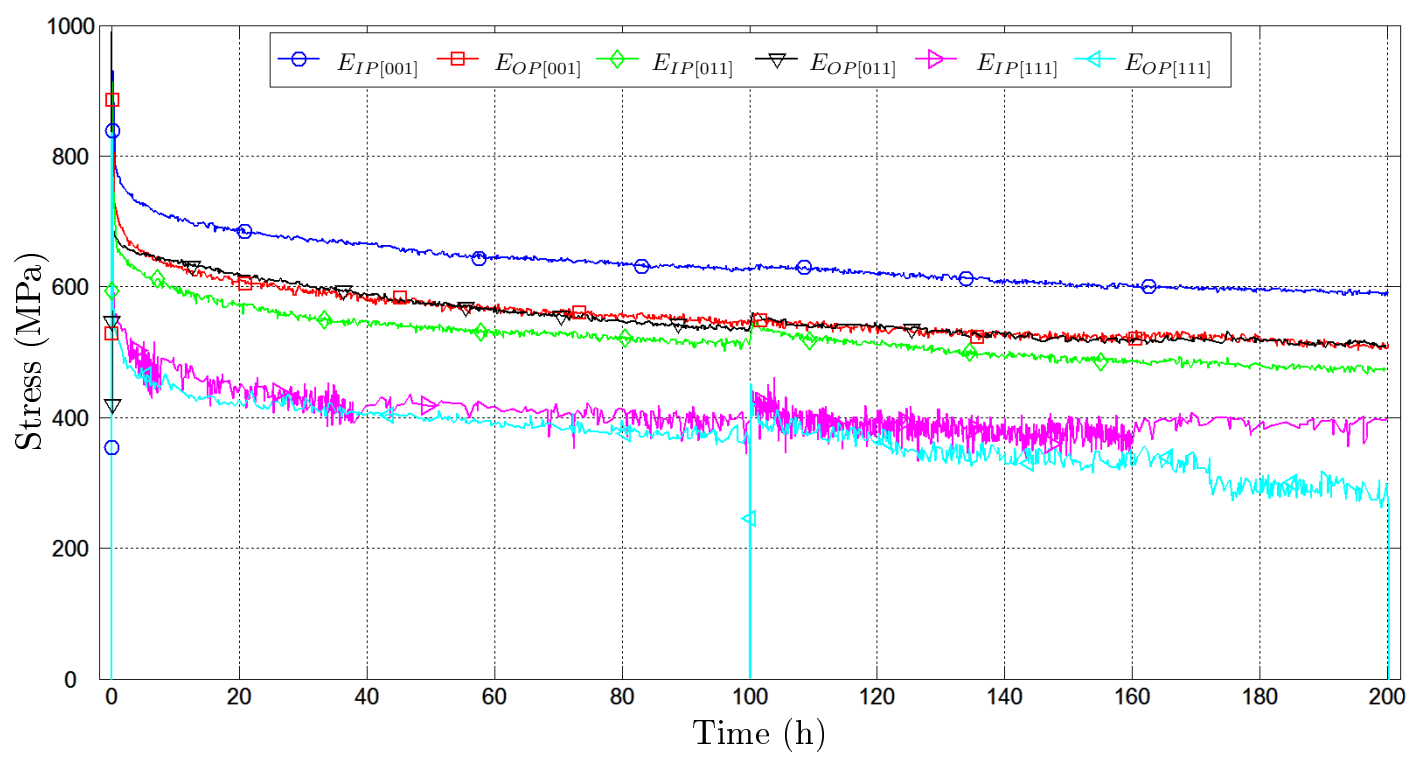

Figure 5: The experimentally obtained stress relaxation curves.

The equivalent stress defined above in Equation (9) is also used, as the observed stress relaxation shows a tension/compression asymmetry, see Figure 5. Thus, by using a simple Norton creep law applied individually on all slip systems the 
following flow function is found

$$
\dot{\lambda}^{\alpha, c r}=C\left(\frac{\sigma_{e}^{\alpha}}{G_{r}}\right)^{n} A(T)
$$

where $C$ and $n$ are parameters describing the creep response (stress relaxation), and where $A(T)$ is an Arrhenius term accounting for the thermal activation

$$
A(T)=A_{0} \mathrm{e}^{-\frac{Q}{R T}}
$$

with the universal gas constant $R=8.3144621 \mathrm{~J} /(\mathrm{mol} \mathrm{K})$, the activation energy $Q$, a constant $A_{0}$ and the temperature $T$ (given in $\mathrm{K}$ ).

By observing the experimental stress relaxation curves found in Figure 5, one can see that the responses are different for the different crystal orientations, thus implying that an anisotropic behaviour is present, which needs to be addressed. The orientation dependence of $C$ has been examined, a more detailed discussion is given in Section 4.1.1, and determined by a projection of the normalised stress state in the crystallographic directions of the $\langle 011\rangle$ and $\langle 111\rangle$ families. The following definition of the anisotropic creep function $C$ is given

$$
C=b_{0}+b_{1} x_{1}\left(\boldsymbol{D}_{S}\right)+b_{2} x_{2}\left(\boldsymbol{D}_{S}\right)
$$

where $b_{0}, b_{1}$ and $b_{2}$ are linear regression parameters determined from the performed parameter optimisation, $\boldsymbol{D}_{S}$ is the normalised stress direction tensor [24], based on the second Piola-Kirchhoff stress tensor $\boldsymbol{S}$ in the following way

$$
D_{S}=\frac{S}{\sqrt{S: S}}
$$

and finally, the $x_{1}\left(\boldsymbol{D}_{S}\right)$ and $x_{2}\left(\boldsymbol{D}_{S}\right)$ are the maximum values of the projections of the normalised stress direction tensor in the crystal directions of the $\langle 011\rangle$ and $\langle 111\rangle$ families (misalignment taken into account) and are defined accordingly to

$$
\left\{\begin{array}{l}
x_{1}\left(\boldsymbol{D}_{S}\right)=\max \left(\langle 011\rangle \boldsymbol{D}_{S}\langle 011\rangle^{T}\right) \\
x_{2}\left(\boldsymbol{D}_{S}\right)=\max \left(\langle 111\rangle \boldsymbol{D}_{S}\langle 111\rangle^{T}\right)
\end{array}\right.
$$

The unique directions which the two crystallographic families consist of are given in Table 2. All of these crystal directions are evaluated, and consequently different values are generated accordingly to the crystallographic orientation of the specimen (FE-model). The maximum value is due to that the crystal direction that gives the largest value is the direction in which the specimen is loaded. The maximum values of the projections quantify the amount of creep flow obtained in the certain crystallographic direction with respect to the stress state. With this above derived approach one is able to predict the creep response in a multiaxial stress state, as well as an uniaxial one, due to that the projections 
Table 2: The crystal directions evaluated in each crystallographic direction family.

\begin{tabular}{l|l}
\hline$\langle 011\rangle$ & {$[011],[011],[110],[110],[101],[101]$} \\
\hline$\langle 111\rangle$ & {$[111],[111],[111],[111]$} \\
\hline
\end{tabular}

yield different values.

The creep exponent $n$ was not considered to follow an anisotropic behaviour, more details regarding this can be found in Section 4.1.1. The creep parameters $b_{0}, b_{1}, b_{2}$ and $n$ were determined through inverse modelling (parameter optimisation) of the experimental stress relaxation curves.

\section{Simulations}

In the simulations of the TMF cycle, only a short section over the middle of the test specimen was modelled and analysed. The reason for this was to obtain the correct constant mechanical strain range over the strain gauge length during the TMF cycle, especially during the long hold-times. The mesh program True Grid ${ }^{\circledR}[25]$ was used to model and mesh the test specimens, taking consideration to the different diameters of the test specimens. The FE-model contains 4800 eight-noded solid brick elements with reduced integration and default hourglass control. As the test specimens are cylindrical a so called butterfly-mesh was adopted to obtain an adequate mapped mesh, the FE-model can be seen in Figure 6. A convergence study with respect to the length of the specimen was performed to see if the anisotropy or if the bending of the specimen influenced the stress-strain state in the model. From this analysis no significant flaws could be observed, and as a consequence the short section was used in the TMF analysis. A virtual extensometer was applied over the strain gauge length, measuring the relative motion of two nodes. The global stress state in the specimens was extracted by taking the total boundary force in the longitudinal direction at the top of the model and divide it with the nominal area of the parallel section in the middle.

The implicit solver in the FE-software LS-DYNA ${ }^{\circledR}$ version 971 [26] was used in the analysis of the experimental TMF cycles. The crystal plasticity and creep behaviour of the material had been implemented into an user-defined material subroutine for the FE-software. The introduced kinematic relations are used to incrementally update the stress state in the body under consideration using a backward-Euler approximation, see e.g. [27], in combination with a NewtonRaphson iterative scheme, in an implicit stress update algorithm. Due to numerical complexities with the crystal plasticity model regarding active slip systems, the initial stiffness method was used, see e.g. [28]. The initial stiffness method means that the elastic tangent stiffness tensor is never updated throughout the 


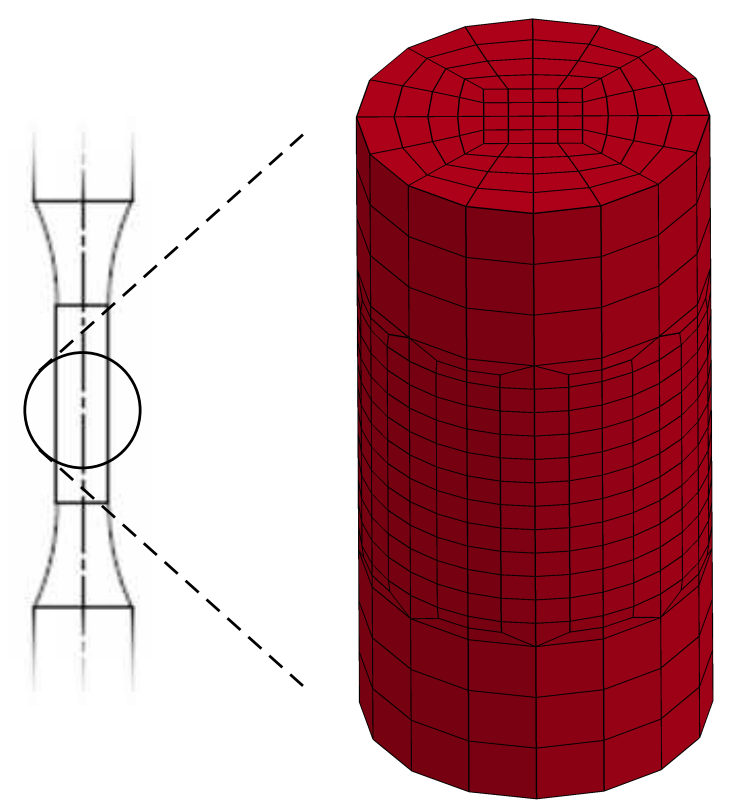

Figure 6: The FE-model of the small straight section of the test specimens used in the simulations showing the mapped butterfly mesh.

Table 3: The elastic constants, units in GPa.

\begin{tabular}{ccccc}
\hline Temp. $\left({ }^{\circ} \mathrm{C}\right)$ & $C_{11}$ & $C_{12}$ & $C_{44}$ & $C_{11}-C_{12}$ \\
\hline 23 & 246.1 & 154.4 & 130.1 & 91.63 \\
208 & 241.0 & 155.9 & 121.1 & 85.13 \\
418 & 231.8 & 152.3 & 113.7 & 79.52 \\
600 & 221.2 & 148.0 & 106.8 & 73.25 \\
700 & 219.9 & 150.4 & 103.0 & 69.56 \\
812 & 212.7 & 147.5 & 98.68 & 59.89 \\
\hline
\end{tabular}

analysis, only the initial stiffness is calculated and used in each of the iterations.

The elastic stiffness is based on the temperature dependent elastic constants found in Table 3, which were determined by resonance measurements. Furthermore, the crystal plasticity-creep model needs the $\kappa$-values and the slip resistance $G_{r}$, as can be found in Table 4 . These were obtained from experimental monotonic tension and compression tests performed at different temperatures and determined as specified in Leidermark et al. [11]. In the FE-simulations interpolations between the different temperatures of the elastic and plastic parameters were used. 
Table 4: The crystal plasticity constants at different temperatures.

\begin{tabular}{ccccccc}
\hline Temp. $\left({ }^{\circ} \mathrm{C}\right)$ & $\kappa_{1}$ & $\kappa_{2}$ & $\kappa_{3}$ & $\kappa_{4}$ & $\kappa_{5}$ & $G_{r}(\mathrm{MPa})$ \\
\hline 20 & 0.27027 & 0.29002 & -0.045656 & 0.001031 & 0.048297 & 541.63 \\
500 & 0.65033 & 0.36121 & -0.0077911 & -0.1163 & 0.082087 & 573.87 \\
750 & 0.44910 & 0.34594 & 0.12689 & -0.23686 & 0.16189 & 514.18 \\
\hline
\end{tabular}

Table 5: The stress relaxation parameters from the curve-fitting.

\begin{tabular}{lcccccc}
\hline Specimen & $E_{I P[001]}$ & $E_{O P[001]}$ & $E_{I P[011]}$ & $E_{O P[011]}$ & $E_{I P[111]}$ & $E_{O P[111]}$ \\
\hline$a_{i}(\mathrm{MPa})$ & 814.67 & 731.16 & 706.55 & 730.50 & 548.16 & 544.03 \\
$b_{i}$ & -0.05926 & -0.06541 & -0.07220 & -0.06530 & -0.07259 & -0.08531 \\
\hline
\end{tabular}

\subsection{Parameter optimisation}

To obtain good values for the parameters concerning the creep behaviour, $b_{0}$, $b_{1}, b_{2}$ and $n$, an optimisation was carried out. In the optimisation a curvemapping operation was performed, in which the calculated response of the stress relaxation from each FE-simulation was compared to the one obtained in the respective experimental test. The optimisation tool LS-OPT ${ }^{\circledR}$ version 4.2 [29] was utilised in the evaluation process of the curve-mapping, with the built-in curve-mapping tool.

\subsubsection{Parameter screening}

The optimisation tool LS-OPT ${ }^{\circledR}$ needs initial values and also maximum and minimum values of each parameter to perform the optimisation. The determination of the initial values to be used in the parameter optimisation are extracted from the experimental tests. This was achieved by performing a screening of the parameters used in the optimisation process. The screening was done in the following way; first the experimental stress relaxation tests from Figure 5 were adopted into general power-law expressions by curve-fitting, only the relaxation part of the stress-time curve was of interest, thus yielding global stress relaxation versus time responses according to

$$
\sigma_{i}=a_{i} t^{b_{i}}
$$

where $i=1,2, \ldots, 6$ is the index of the respective test, values of $a_{i}$ and $b_{i}$ can be found in Table 5 .

The next step was to evaluate the stress relaxation rate, thus the above equations were differentiated with respect to the time

$$
\dot{\sigma}_{i}=a_{i} b_{i} t^{b_{i}-1}
$$


Table 6: The elastic stiffness for each of the three nominal crystallographic orientations, units in GPa.

\begin{tabular}{ccc}
\hline$E_{[001]}$ & $E_{[011]}$ & $E_{[111]}$ \\
\hline 95 & 170 & 254 \\
\hline
\end{tabular}

This was done so that the global creep strain rates could be determined from the additively decomposed total strain rate, where the total strain is $\varepsilon^{t o t}=\varepsilon^{e}+\varepsilon^{c r}$. As the stress relaxation tests were conducted at a constant strain range, hence the differentiated equation with respect to time of the total strain is

$$
\dot{\varepsilon}^{t o t}=\dot{\varepsilon}^{e}+\dot{\varepsilon}^{c r}=0 \Rightarrow \dot{\varepsilon}^{c r}=-\dot{\varepsilon}^{e}
$$

and taking into account that $\varepsilon^{e}=\sigma / E$, the following relationship is obtained

$$
\dot{\varepsilon}^{c r}=-\frac{\dot{\sigma}}{E}
$$

This method was used by Woodford [30] who derived an alternative approach to evaluate constant load creep tests by using constant strain tests (TMF cycling) on the basis of the work done by Hart [31]. Furthermore, in the work of Segersäll et al. [20] it was shown how this method could be applied to a single-crystal superalloy undergoing TMF stress relaxation. The obtained creep strain rates were compared to regular uniaxial creep experiments with satisfying agreement.

At three stress states in each of the six tests the global creep strain rates were evaluated by the above described procedure, using the elastic stiffnesses according to the ones stated in Table 6 . The three evaluated states of the TMF stress relaxation curve are:

1) the first onset of stress relaxation (start of the first hold-time),

2) the region in the first hold-time, where the stress relaxation curve has a pronounced slope change (the bend of the curve), and

3) the very end of the stress relaxation (end of the second hold-time).

A more detailed overview of the positions can be found in Figure 7. After the creep strain rate evaluation from the stress relaxation tests an analytical calculation in MATLAB ${ }^{\circledR}$ [32] was performed. The extracted global creep strain rates and stresses were then projected down on the crystallographic slip systems, to determine the amount of creep shear strain rate $\dot{\gamma}^{\alpha, c r}$ and equivalent stress $\sigma_{e}^{\alpha}$, i.e. the crystallographic equivalence of the global stress relaxation response. When these crystallographic entities were known for each test the parameters in the crystallographic Norton creep law could be established. A curve-fitting procedure for each crystal orientation and load case was done taking consideration to the misalignment in orientation of the real test specimens, see Figure 8. The determined constants are stated in Table 7 and defined accordingly as 
in Equation (14). As can be seen in Figure 8 the slopes of the curves are more or less the same, which constitutes an isotropic creep exponent $n$. Furthermore, one can observe that the positions of the interposing curves are different, which leads to that the creep function $C$ is dependent on orientation, thus anisotropic. Due to this, the $C$ function is given the anisotropic form as stated in Equation (16).

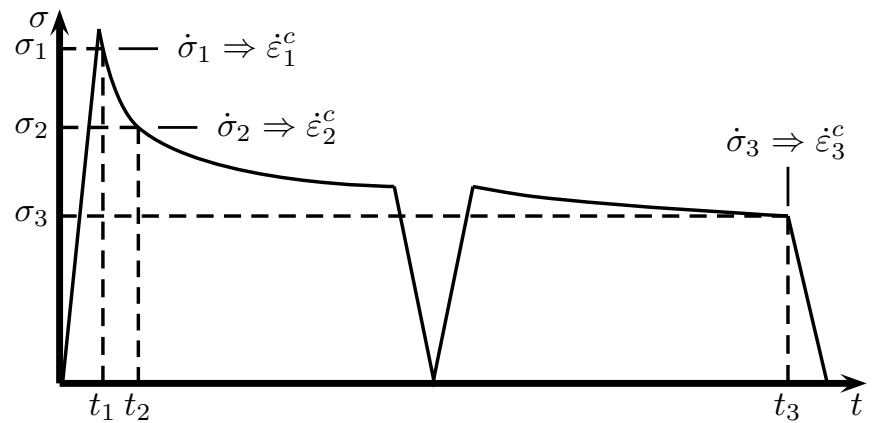

Figure 7: The schematic view of the evaluation points of the creep strain rates and corresponding stresses and stress rates.

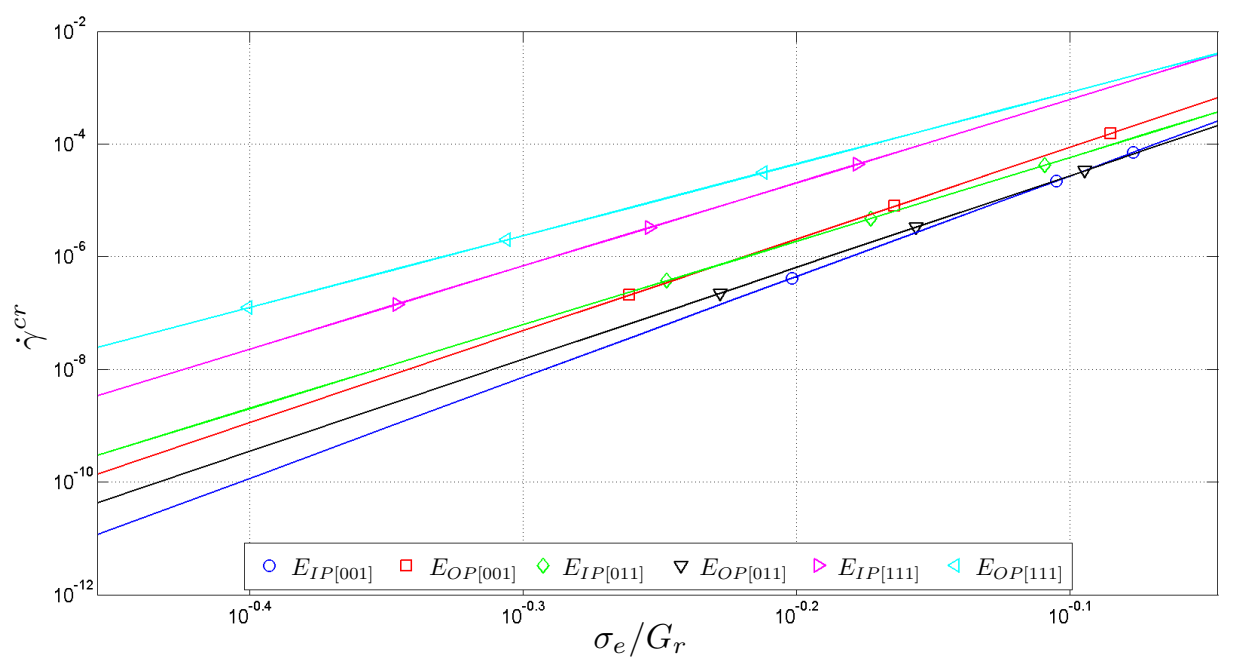

Figure 8: The experienced crystallographic creep shear strain rate versus the equivalent stress divided by the slip resistance in the six experiments.

To determine the initial values of $b_{0}, b_{1}$ and $b_{2}$ in the expression of the creep function $C$, the following least square regression model was set up based on the fitted values

$$
\boldsymbol{X} \boldsymbol{b}=\boldsymbol{C}
$$

and in more detail, with all the specific entities specified, the equation system 
Table 7: The creep flow constants determined for each crystal orientation and load case

\begin{tabular}{cccccc}
\hline$C_{I P[001]}$ & $C_{I P[011]}$ & $C_{I P[111]}$ & $C_{O P[001]}$ & $C_{O P[011]}$ & $C_{O P[111]}$ \\
\hline 0.0016633 & 0.0037115 & 0.0017528 & 0.0011747 & 0.018461 & 0.015354 \\
\hline$n_{I P[001]}$ & $n_{I P[011]}$ & $n_{I P[111]}$ & $n_{O P[001]}$ & $n_{O P[011]}$ & $n_{O P[111]}$ \\
\hline 17.88 & 14.85 & 14.78 & 16.29 & 16.31 & 12.72 \\
\hline
\end{tabular}

Table 8: The initial values and the minimum and maximum values obtained from the confidence interval for the creep parameters.

\begin{tabular}{lcccc}
\hline & $b_{0}$ & $b_{1}$ & $b_{2}$ & $n$ \\
\hline initial & 0.0018723 & -0.0204587 & 0.0300536 & 15.4717 \\
minimum & -0.0086001 & -0.0339413 & 0.0198531 & 12.0087 \\
maximum & 0.0123448 & -0.0069762 & 0.0402540 & 18.9347 \\
\hline
\end{tabular}

to be solved is

$$
\left[\begin{array}{lll}
1 & x_{1, I P[001]} & x_{2, I P[001]} \\
1 & x_{1, I P[011]} & x_{2, I P[011]} \\
1 & x_{1, I P[111]} & x_{2, I P[111]} \\
1 & x_{1, O P[001]} & x_{2, O P[001]} \\
1 & x_{1, O P[011]} & x_{2, O P[011]} \\
1 & x_{1, O P[111]} & x_{2, O P[111]}
\end{array}\right]\left[\begin{array}{l}
b_{0} \\
b_{1} \\
b_{2}
\end{array}\right]=\left[\begin{array}{l}
C_{I P[001]} \\
C_{I P[011]} \\
C_{I P[111]} \\
C_{O P[001]} \\
C_{O P[011]} \\
C_{O P[111]}
\end{array}\right]
$$

By solving the equation system above, using the least square method, the initial values are received, see Table 8 .

As stated previously, LS-OPT ${ }^{\circledR}$ requires the minimum and maximum value of each parameter to perform an optimisation, thus a confidence interval for the regression coefficients $\bar{b}_{i}$ is set up according to e.g. Myers et al. [33]. A $95 \%(100(1-\psi) \%)$ confidence interval following normal $t$-statistics with $d o f=6-3=3$ degrees of freedom has the following appearance

$$
b_{i}-t_{\psi / 2, \operatorname{dof}} \sqrt{\hat{\sigma}^{2}\left(\boldsymbol{X}^{T} \boldsymbol{X}\right)_{i i}^{-1}} \leq \bar{b}_{i} \leq b_{i}+t_{\psi / 2, \operatorname{dof}} \sqrt{\hat{\sigma}^{2}\left(\boldsymbol{X}^{T} \boldsymbol{X}\right)_{i i}^{-1}}
$$

where $t_{\psi / 2=0.025, \text { dof }=3}=3.182$ is the $t$ distribution, $\hat{\sigma}^{2}=\left(\boldsymbol{C}^{T} \boldsymbol{C}-\boldsymbol{b}^{T} \boldsymbol{X}^{T} \boldsymbol{C}\right) /$ dof is the estimate of the error variance, and where $\left(\boldsymbol{X}^{T} \boldsymbol{X}\right)_{i i}^{-1}$ is the $(i, i)$ th element of the matrix $\left(\boldsymbol{X}^{T} \boldsymbol{X}\right)^{-1}$. The maximum and minimum value obtained from the confidence interval for each parameter can be found in Table 8 . In the table one can see that the minimum of $b_{0}$ is negative. If combined with the others parameters in a certain way during an optimisation step the total of $C$ might also become negative, this is not a desired property. To account for this during the optimisation a MacCauly bracket is used for the expression of $C$, according to

$$
C=\left\langle b_{0}+b_{1} x_{1}\left(\boldsymbol{D}_{S}\right)+b_{2} x_{2}\left(\boldsymbol{D}_{S}\right)\right\rangle
$$


If $C$ would become negative then $C=0$ and a poor correlation with the experimental TMF stress relaxation is obtained, thus the evaluation values for the parameters in the meta-model of the optimisation step in question are considered bad estimates.

Regarding the creep exponent $n$ (slope of the curve), which does show a little difference between the crystal orientations, but the outcome of changing the exponent for the different the crystal orientations is negligible small. Based on this it is thus motivated to use a normal mean value of the $n$ parameter between the orientations as an initial value according to

$$
\bar{n}=\frac{1}{N} \sum_{i=1}^{N} n_{i}
$$

Inserting the values found in Table 7 yields a mean value of $\bar{n}=15.4717$. The maximum and minimum value of the creep exponent are determined by using a corrected sample standard deviation

$$
s=\sqrt{\frac{1}{N-1} \sum_{i=1}^{N}\left(n_{i}-\bar{n}\right)^{2}}
$$

and a $95 \%$ confidence interval which corresponds to 1.959964 standard deviations, thus the values are derived from

$$
\bar{n}-1.959964 s \leq \bar{n} \leq \bar{n}+1.959964 s
$$

leading to the minimum and maximum values of 12.0087 and 18.9347 respectively, a summary can be found in Table 8 .

\subsubsection{Computational aspects of the optimisation}

Due to the number of variables and that different crystal orientations at both IP and OP TMF were analysed, the parameter optimisation was conducted using a meta-model based on a radial basis function network with space filling, containing 294 evaluation points in total. This number of evaluations are based on the following: each load case (2) contains three crystal orientations (3) which are to be evaluated at eight simulation points (8) per iteration, the number of iterations are set to six $(6)$, plus one verification run $(2 \cdot 3)$, hence; $2 \cdot 3 \cdot 8 \cdot 6+2 \cdot 3=294 \mathrm{FE}$-simulations were carried out.

To handle the large amount of FE-simulations in the optimisation a large Linuxbased cluster at the National Supercomputer Centre (NSC) [34] in Linköping, Sweden, was used. The Linux-based cluster, named Kappa, contains 364 HP ProLiant DL170h G6 computational servers and two HP proliant DL980 G7 
Table 9: The optimised values of the creep parameters.

\begin{tabular}{cccc}
\hline$b_{0}$ & $b_{1}$ & $b_{2}$ & $n$ \\
\hline-0.00224667 & -0.022414 & 0.040254 & 12.0087 \\
\hline
\end{tabular}

huge nodes with large amounts of memory, hence giving a combined peak performance of 27 TFLOPS. The computational servers are equipped with two Intel Xeon E5520 quad-core processors with 24 or 72 GB RAM each. The huge nodes have 1 TiB RAM and eight Intel Xeon E7-2800 processors with eight cores each, giving 64 cores in total. Thus, in total Kappa has 3040 computational cores. The computational nodes communicate with each other over a high-speed network of InfiniBand.

Each of the FE-simulations in the parameter optimisation was performed using eight cores on a computational server, where the average time for a simulation took roughly 1.3 clock hours.

\subsection{Thermally activated process}

The thermally activated creep process during the TMF cycle is handled by the Arrhenius term in the crystallographic Norton creep law. The Arrhenius term is disregarded in the evaluation process of the above derived creep parameters as the temperature is held constant during the TMF stress relaxation (long hold-time). The parameters in the creep expression were defined at $750^{\circ} \mathrm{C}$ and due to this fact, the Arrhenius term is to yield unity at $750^{\circ} \mathrm{C}$ to achieve the correct evaluated values. The constants in the Arrhenius term are defined by taking the logarithm of the expression and performing a regression using creep strain rates from uniaxial creep tests at $T_{1}=700^{\circ} \mathrm{C}$ and $T_{2}=750^{\circ} \mathrm{C}$ with the same stress state and normalising the creep strain rate around $750^{\circ} \mathrm{C}$ of the logarithmic expression

$$
\left[\begin{array}{cc}
1 & -\frac{1}{R T_{1}} \\
1 & -\frac{1}{R T_{2}}
\end{array}\right]\left[\begin{array}{c}
\ln A_{0} \\
Q
\end{array}\right]=\left[\begin{array}{l}
\ln A\left(T_{1}\right) \\
\ln A\left(T_{2}\right)
\end{array}\right]
$$

with $A\left(700^{\circ} \mathrm{C}\right)=0.20212$ and $A\left(750^{\circ} \mathrm{C}\right)=1$ yielding $Q=264730 \mathrm{~J} / \mathrm{mol}$ and $A_{0}=3.273 \cdot 10^{13}$.

\section{Results and discussion}

From the above performed parameter optimisation the creep parameters were determined, and the obtained TMF stress relaxation behaviour can be seen in Figure 9 with the optimised values as stated in Table 9. When comparing the differences between the simulated and experimentally obtained stress relaxation curves, one can see a good correlation. It can be noted that during the second 
hold-time in the TMF cycle of the two simulated [111] crystal orientations the correlation is slightly decreased. In these cases, the second loading in the TMF cycle yields much higher stress compared to the other orientations relative to the relaxed stress state of the first hold-time. One reason for this behaviour can be, as the [111] crystal orientation is the stiffest one, that an internal numerical stiffness is present in the analyses. This behaviour needs further investigations to be handled, so that a better correlation can be reached. Though, eventually the stress state of the two [111] crystal orientations relaxes down to the experimental level in the end of the second hold-time.

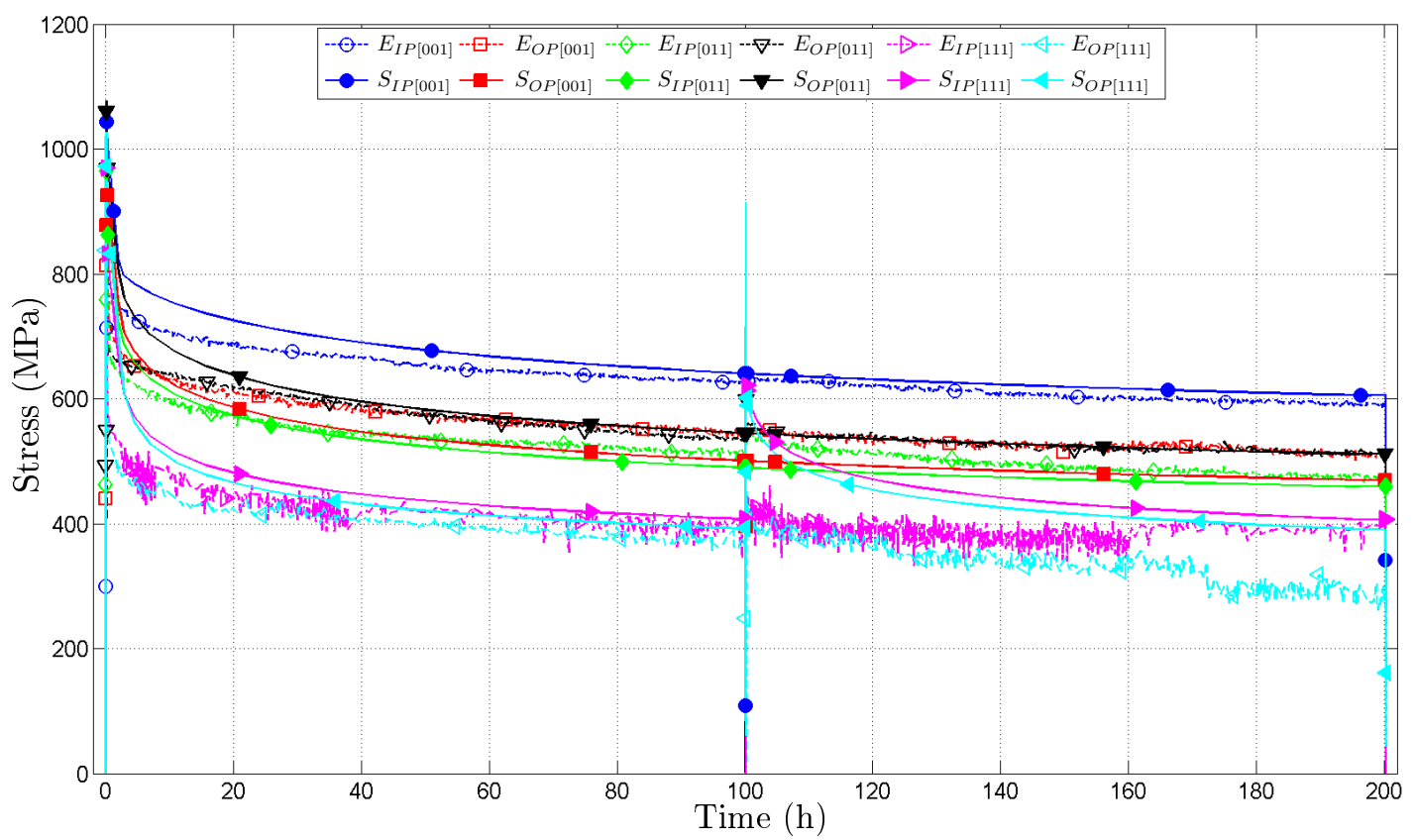

Figure 9: The TMF stress relaxation curves from the simulations compared to the experimentally obtained ones ( $E$ - experiment and $S$ - simulation).

Regarding the anomalous plastic behaviour, one can see that the anisotropy and the relative tension/compression asymmetry for the respective crystal orientations are present. The pronounced tension/compression asymmetry for the [001] crystal orientation, where the IP case leads to a higher stress state than the OP case, is accounted for and so is also the less pronounced opposite asymmetry in the [011] orientation and the small difference in the [111] orientation. The combination of the equivalent stresses $\sigma_{e}^{\alpha}$ with the anisotropic creep expression manage to describe the experimentally observed asymmetry in tension (IP) and compression (OP) as well as the anisotropy with satisfying results.

In general the experiments in the [111] crystal orientations were more demanding than the other two crystal orientations. This can be observed in Figure 5, 
where the experimental curves experience a lot of disturbance. A reason for this might be the high stiffness in the [111] orientation and the disturbance should be referred to instabilities in the TMF testing machine and not the microstructure. The dip of the experimental OP [111] at the end of the second hold-time $(t \approx 170 \mathrm{~h}$ ) might be due to experimental problems of the TMF testing machine and is not to be misunderstood as a real material behaviour. As we can see that the behaviour of the stress relaxation continues as before, but with a translation in stress level.

Concerning the parameter optimisation in LS-OPT, 6 iterations and a verification run were performed. When taking a look at the received result of the optimised values of the creep parameters in the verification run, the multiobjective was equal to 0.319. A detailed overview of all the optimisation steps was performed and a better response was found in the optimisation step 4.1 , with a multiobjective of 0.267 . Thus yielding the optimised set of parameters which is considered as the final result of the optimisation. A possibility during an optimisation step is that the estimate can be worse than in the previous step, which is unexpected as the result should become more optimal or yield the same value with increasing optimisation steps. In the optimisation process, the program is searching for a global optimum, but it might end up in a local optimum. From the result of the verification run we see that it is not as good as the one in the optimisation step 4.1, thus indicating a local optimum and the global optimum of the investigated interval is found in step 4.1 .

On the basis of the above mentioned optimisation, another interesting thing to point out is that the optimised value for the creep exponent $n$ is equal to the minimum value obtained in the screening process. This sets pressure on the rightfulness of the screening, could it have been performed in an other way or is it satisfying. As the optimised value of $n$ is found to be one of the limits it is likely that some more considerations have to be made. The limits are not physically determined and a thought is to let the creep exponent be freely determined in the optimisation, hence no limits would be set. In the parameter screening process the limits for the parameters $b_{0}, b_{1}$ and $b_{2}$ are defined by a $95 \%$ confidence interval and this method has also been used for the creep exponent $n$. Thus, from the above, one can motivate the procedure from a consistently point of view that the same approach has been used in the two screening procedures.

\section{Conclusions}

- A model for the plastic and creep behaviour of the single-crystal nickelbase superalloy MD2 has been set up, where the anisotropy and tension/compression asymmetry are accounted for. 
- The developed model is able to describe the anisotropic stress relaxation behaviour of the material in question with good correlation to the performed TMF experiments.

- The question of finding the true global optimal solution for the creep parameters is a matter for further work, even though, as mentioned above, the found one gives a satisfactory result.

\section{Acknowledgements}

The work has been financially supported by Siemens Industrial Turbomachinery AB in Finspång, Sweden, and the Swedish Energy Agency, via the Research Consortium of Materials Technology for Thermal Energy Processes, Grant No. KME-502. The use of the well equipped Linux-based cluster Kappa at the National Supercomputer Centre in Linköping, Sweden, is also greatly acknowledged. Productive discussions with Professor K. Simonsson and Professor J.J. Moverare, at Linköping University, are greatly acknowledged.

\section{References}

[1] K. Dietrich, J.M. Latorre, L. Olmos, A. Ramos, The role of flexible demands in smart energy systems, in: V. Pappu, M. Carvalho, P. Pardalos (Eds.), Optimization and Security Challenges in Smart Power Grids, 2013, pp. 79-97.

[2] C.R. Reed, The Superalloys - Fundamentals and Applications, Cambridge University Press, Cambridge, 2006.

[3] A. Pineau, S.D. Antolovich, High temperature fatigue of nickel-base superalloys - A review with special emphasis on deformation modes and oxidation, Engineering Failure Analysis, 16, (2009), 2668-2697.

[4] D.R. Askeland, The Science and Engineering of Material, Nelson Thornes Ltd, Cheltenham, 2001.

[5] P. Haupt, Continuum Mechanics and Theory of Materials, Springer-Verlag, Berlin, 2002.

[6] F. Roters, P. Eisenlohr, L. Hantcherli, D.D. Tjahjanto, T.R. Bieler, D. Raabe, Overview of constitutive laws, kinematics, homogenization and multiscale methods in crystal plasticity finite-element modeling: Theory, experiment, applications, Acta Materialia, 58, (2010), 1152-1211.

[7] G.M. Han, J.J. Yu, X.F. Sun, Z.Q. Hu, Thermo-mechanical fatigue behavior of a single crystal nickel-based superalloy, Materials Science and Engineering A, 528, (2011), 6217-6224. 
[8] J.J. Moverare, S. Johansson, R.C. Reed, Deformation and damage mechanisms during thermal-mechanical fatigue of a single-crystal superalloy, Acta Materialia, 57, (2009), 2266-2276.

[9] Q. Qin, J.L. Bassani, Non-Schmid yield behavior in single crystals, J. Mech. Phys. Solids, 40, No. 4, (1992), 813-833.

[10] Q. Qin, J.L. Bassani, Non-associated plastic flow in single crystals, J. Mech. Phys. Solids, 40, No. 4, (1992), 835-862.

[11] D. Leidermark, J.J. Moverare, K. Simonsson, S. Sjöström, S. Johansson, Room temperature yield behaviour of a single-crystal nickel-base superalloy with tension/compression asymmetry, Computational Materials Science, 47, (2009), 366-372.

[12] A. Ma, D. Dye, R.C. Reed, A model for the creep deformation behaviour of single-crystal superalloy CMSX-4, Acta Materialia, 56, (2008), 1657-1670.

[13] Z. Zhu, H. Basoalto, N. Warnken, R.C. Reed, A model for creep deformation behaviour of nickel-based single crystal superalloys, Acta Materialia, 60, (2012), 4888-4900.

[14] A. Staroselsky, B.N. Cassenti, Creep, plasticity, and fatigue of single crystal superalloy, International Journal of Solids and Structures, 48, (2011), 20602075.

[15] R.N. Ghosh, R.V. Curtis, M. McLean, Creep deformation of single crystal superalloys - Modelling the crystallographic anisotropy, Acta Metallurgica et Materialia, 38, No. 10, (1990), 1977-1992.

[16] I.N. Vladimirov, S. Reese, G. Eggeler, Constitutive modelling of the anisotropic creep behaviour of nickel-base single crystal superalloys, International Journal of Mechanical Sciences, 51, (2009), 305-313.

[17] D.W. MacLachlan, L.W. Wright, S. Gunturi, D.M. Knowles, Constitutive modelling of anisotropic creep deformation in single crystal blade alloys SRR99 and CMSX-4, International Journal of Plasticity, 17, (2001), 441467.

[18] A. Epishin, T. Link, M. Nazmy, M. Staubli, H. Klingelhöffe, G. Holze, Microstructural degradagtion of CMSX-4: kinetics and effects on mechanical properties. in: R.C. Reed, K.A. Green, P. Caron, T.P. Gabb, M.G. Fahrmann, E.S. Huron, et al. (Eds.), Superalloys 2008, Warrendale (PA): The Minerals, Metals \& Materials Society, 2008, pp. 725-731.

[19] D. Leidermark, J.J. Moverare, S. Johansson, K. Simonsson, S. Sjöström, Tension/compression asymmetry of a single-crystal superalloy in virgin and degraded condition, Acta Materialia, 58, (2010), 4986-4997. 
[20] M. Segersäll, J. Moverare, D. Leidermark, K. Simonsson, Creep and stress relaxation anisotropy of a single-crystal superalloy, Metallurgical and $\mathrm{Ma}$ terials Transactions A, doi: 10.1007/s11661-014-2198-0, (2014).

[21] E.H. Lee, Elastic-plastic deformation at finite strains, Journal of Applied Mechanics, 36, No. 1, (1969), 1-6.

[22] D. Peirce, R.J. Asaro, A. Needleman, An analysis of nonuniform and localized deformation in ductile single crystals, Acta Metallurgica, 30, No. 6, (1982), 1087-1119.

[23] D.P. Pope, S.S. Ezz, Mechanical properties of $\mathrm{Ni}_{3} \mathrm{Al}$ and nickel-base alloys with high volume fraction of $\gamma^{\prime}$, International Metals Reviews, 29, No. 3, (1984), 136-167

[24] R. Mücke, O-E. Bernhardi, A constitutive model for anisotropic materials based on Neuber's rule, Computer Methods in Applied Mechanics and Engineering, 192, No. 37-38, (2003), 4237-4255.

[25] R. Rainsberger, TrueGrid ${ }^{\circledR}$ User's Manual, XYZ Scientific Applications Inc., Livermore, 2006.

[26] J.O. Hallquist, LS-DYNA ${ }^{\circledR}$ Theory Manual, Livermore Software Technology Corporation, Livermore, 2006.

[27] T. Belytschko, W.K. Liu, B. Moran, Nonlinear Finite Elements for Continua and Structures, John Wiley \& Sons Ltd., Chichester, 2000.

[28] O.O.R. Famiyesin, Robust symmetric formulations for nonassociated plasticity problems, Journal of Engineering Mechanics, 125, No. 9, (1999), 1071-1080.

[29] N. Stander, W. Roux, T. Goel, T. Eggleston, K. Craig, LS-OPT ${ }^{\circledR}$ User's Manual, Livermore Software Technology Corporation, Livermore, 2010.

[30] D.A. Woodford, Test methods for accelerated development, design and life assesment of high-temperature materials, Materials $\& 3$ Design, 14, No. 4, (1993), 231-242.

[31] E.W. Hart, Constitutive relations for the nonelastic deformation of metals, Journal of Engineering Materials and Technology, (1976), 193-202.

[32] MATLAB version 7.12.0.635 (R2011a), The MathWorks Inc., Natick, www.mathworks.com, 2014-01-08.

[33] R.H. Myers, D.C. Montgomery, C.M. Anderson-Cook, Response Surface Methodology - Process and Product Optimization Using Designed Experiments, John Wiley \& Sons Inc., Hoboken, 2009.

[34] National Supercomputer Centre (NSC), Linköping, Sweden, www.nsc.liu.se, 2014-01-09. 